\title{
Report on upgrade of NIS magnetic observatories by the INTAS infrastructure action CRENEGON
}

\author{
J. L. Rasson ${ }^{1}$, A. S. Potapov ${ }^{2}$, V. E. Korepanov ${ }^{3}$, S. Y. Khomutov ${ }^{4}$, V. M. Krasnov ${ }^{5}$, and J. Bitterly ${ }^{6}$ \\ ${ }^{1}$ Institut Royal Météorologique, CPG, B-5670 Dourbes, Belgium \\ ${ }^{2}$ Institute of Solar-Terrestrial Physics SD RAS, Irkutsk 664033, Russia \\ ${ }^{3}$ Lviv Center of Institute of Space Research, Lviv 79000, Ukraine \\ ${ }^{4}$ Geophysical Observatory "Klyuchi”, Novosibirsk 630090, Russia \\ ${ }^{5}$ Institute of Ionosphere of Ministry of Education and Sciences of the Republic of Kazakhstan, Kazakhstan 480020 \\ ${ }^{6}$ Observatoire Magnétique National, Chambon-la-Forêt 45340, France
}

(Received April 25, 2005; Revised August 23, 2005; Accepted August 26, 2005; Online published June 2, 2006)

\begin{abstract}
We present the overall strategy used to upgrade 9 former USSR observatories to INTERMAGNET standards. The following aspects are discussed: application for funding to the INTAS European ad-hoc agency, choice of observatories to upgrade, choice of the instrumentation to purchase and to install, calibration of the instrumentation and used standards, back-ups, installation of the instrumentation and observatory real estate upgrade, acquisition and communication, training of the observers and documentation, evaluation of the upgrades and management of the project. Several observatories were successfully upgraded and we expect their INTERMAGNET certification soon.
\end{abstract}

Key words: Observatory, geomagnetic observations, NIS science, New Independent States, Russia, Kazakhstan, Ukraine, INTAS, DIflux, magnetometer, standard.

\section{Introduction}

INTAS is a funding body created by the EU for fostering scientific and technological development between the New Independent States (NIS) who made up the Soviet Union and the EU. INTAS has a program for funding Infrastructure Actions intended to renovate the infrastructure of scientific facilities in NIS. We initiated the project CRENEGON (The Creation of a Renewed Network of Basic Geomagnetic Observatories of NIS Countries) in the framework of this program with the aim of improving magnetic observatories considered here as essential scientific infrastructure.

The first presentation of the project was made by Alex Potapov \& Valery Korepanov to the OPSCOM at the 1999 INTERMAGNET meeting in Budapest. It was improved during the 2000 Hurbanovo workshop in several discussions with Ole Rasmussen and Jacques Bitterly. A few months later IPGParis decided to join the project by planning the upgrade of the Borok observatory. The funding success was announced at the 2002 Hermanus magnetic observatories Workshop. Table 1 gives the various amendments required by INTAS during the discussions following the acceptance of the project.

The project was prepared and run by a consortium including the authors and the following institutes:

- Institut de Physique du Globe, Paris, France

- Quantum Magnetometry Laboratory, Ural State Technical University, Ekaterinburg, Russia

Copyright (c) The Society of Geomagnetism and Earth, Planetary and Space Sciences (SGEPSS); The Seismological Society of Japan; The Volcanological Society of Japan; The Geodetic Society of Japan; The Japanese Society for Planetary Sciences; TERRAPUB.
- Earth's and Planetary Magnetism Division, IZMIRAN, Moscow region, Russia

- Siberian Branch of Russian Academy of Sciences

- Institute of Geophysics, Ukrainian Academy of Sciences

Our idea was to get funding from other sources also, trying thus to reach the original goal of 9 upgraded observatories in the NIS. Other funding and support originated from Institut de Physique du Globe de Paris (IPGParis), France and Belsk observatory, Poland.

\section{The Infrastructure Action}

\subsection{Means made available by INTAS}

It was agreed with INTAS that we would get the funds for modernizing and equipping 2 observatories (IRT, AAA) plus 2 complete sets of equipment which would be intended as back-up devices. Part-time salaries for 2 observatories (11 people) for the duration of project $(1.5 \mathrm{y})$, travel funds for meetings and installation, calibration, training and coordination visits, funds for repair and upgrades in observatory buildings, funds for consumables and fluids (isp's) during the project activity.

\subsection{Aims of CRENEGON}

The aims of the project CRENEGON were to modernize in a lasting way:

a) Infrastructure: buildings, instrumentation, telecoms, data acquisition equipment,

b) data processing and observation techniques,

c) ... even salaries of selected geomagnetic observatories in NIS. 


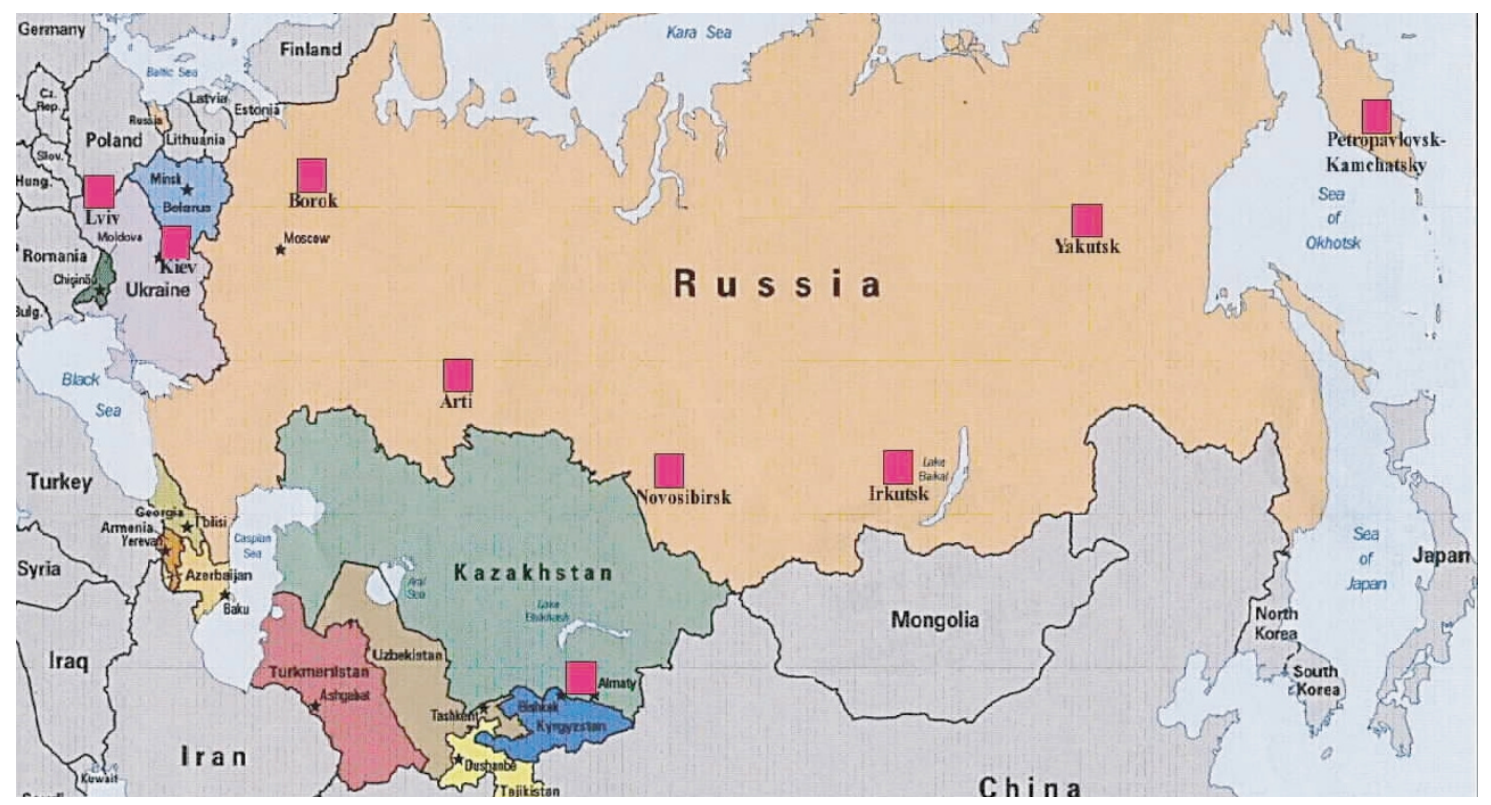

Fig. 1. Magnetic observatories targeted by the CRENEGON INTAS infrastructure action.

Table 1. Applications for funding to the INTAS European ad-hoc Agency.

\begin{tabular}{lllc}
\hline \multicolumn{1}{c}{ Application } & Date & \multicolumn{1}{c}{ Number of Obs } & Requested funding \\
\hline 1st CRENEGON proposal & Nov 2000 & 10 obs & $275 \mathrm{k}$ \\
Rev1 & Dec 2000 & 9 obs & $240 \mathrm{k}$ \\
CRENEGON Light & Apr 2001 & 5 obs & $118 \mathrm{k}$ \\
CRENEGON XXS & Oct 2001 & 2 obs & $57 \mathrm{k}$ \\
CRENEGON V4 & Feb 2002 & 2 obs +2 back-ups & $111 \mathrm{k}$ \\
\hline
\end{tabular}

By "in a lasting way" we understood that there was to be an agreement for a contractual period of 10 years for the action when the host Institutes would provide the same level of salaries and running costs as provided by the Action. For what concerns the quality of the observations and measurements performed at the observatories, we agreed that reaching the INTERMAGNET standards would be our goal.

\subsection{Choice of NIS observatories to upgrade}

Choice was first motivated by an optimal geographic distribution: filling in the gaps in observatory distribution. Another factor was the availability of dedicated staff willing to upgrade. Considering those criteria, a tentative list of the targeted observatories was established: IRT; AAA; NVS; KIV (with IPGParis); PET; BOX (IPGParis); LVV (with the help of Belsk observatory).

\section{Implementation of the Project}

Figure 2 gives the team flow for performing the different tasks of the various teams.

\subsection{Selecting the instruments}

It was decided to purchase NIS manufactured magnetometers for the upgrade of the observatories in the infrastructure action. The motivation for this was:

- Availability inside NIS of "state of the art" devices, known to us by tests before the start of the project.

- Local manufacturers would facilitate the supply, oper- ation and servicing of the magnetometers.

- Cost reduction due to competitive pricing and import tax reductions were to be expected.

- Benefit to the NIS even more by supporting NIS manufacturers and vendors.

Basically we had to equip the observatories with:

- Digitally recording 3-component variation geomagnetometer (variometer).

- Theodolite mounted single axis magnetometer (DIflux).

- Proton magnetometer for measuring the modulus of the geomagnetic field (scalar magnetometer).

- Data loggers.

- Internet hardware for transmission to INTERMAGNET Geomagnetic Information Nodes (GIN).

- Computers for data computation and reduction.

We felt those equipment needs could be covered by purchasing the following devices:

* Fluxgate variometer LEMI-008.

* DIflux LEMI-203.

* Overhauser Proton magnetometer POS-1.

* Personal Computer with Serial ports and Telephone line Modem.

Instrumentation was installed by the partner/manufacturer 


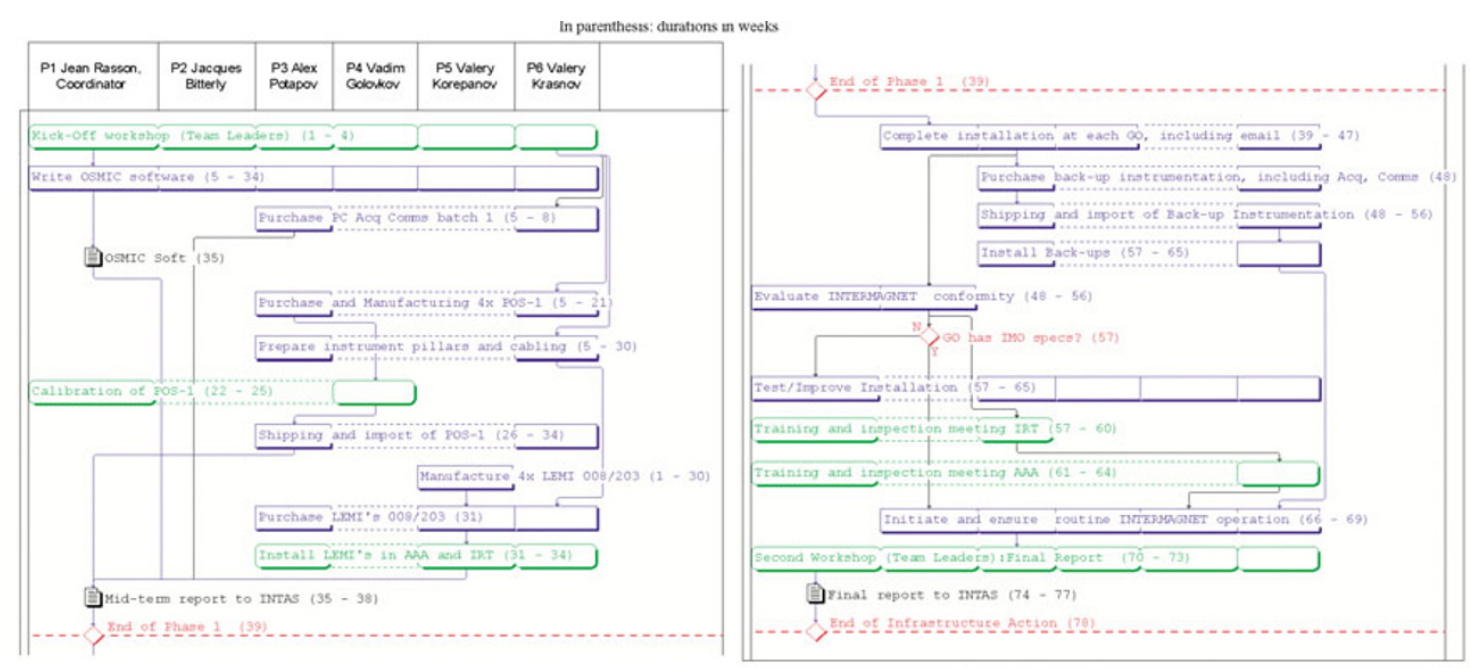

Fig. 2. Team flow of the infrastructure action.
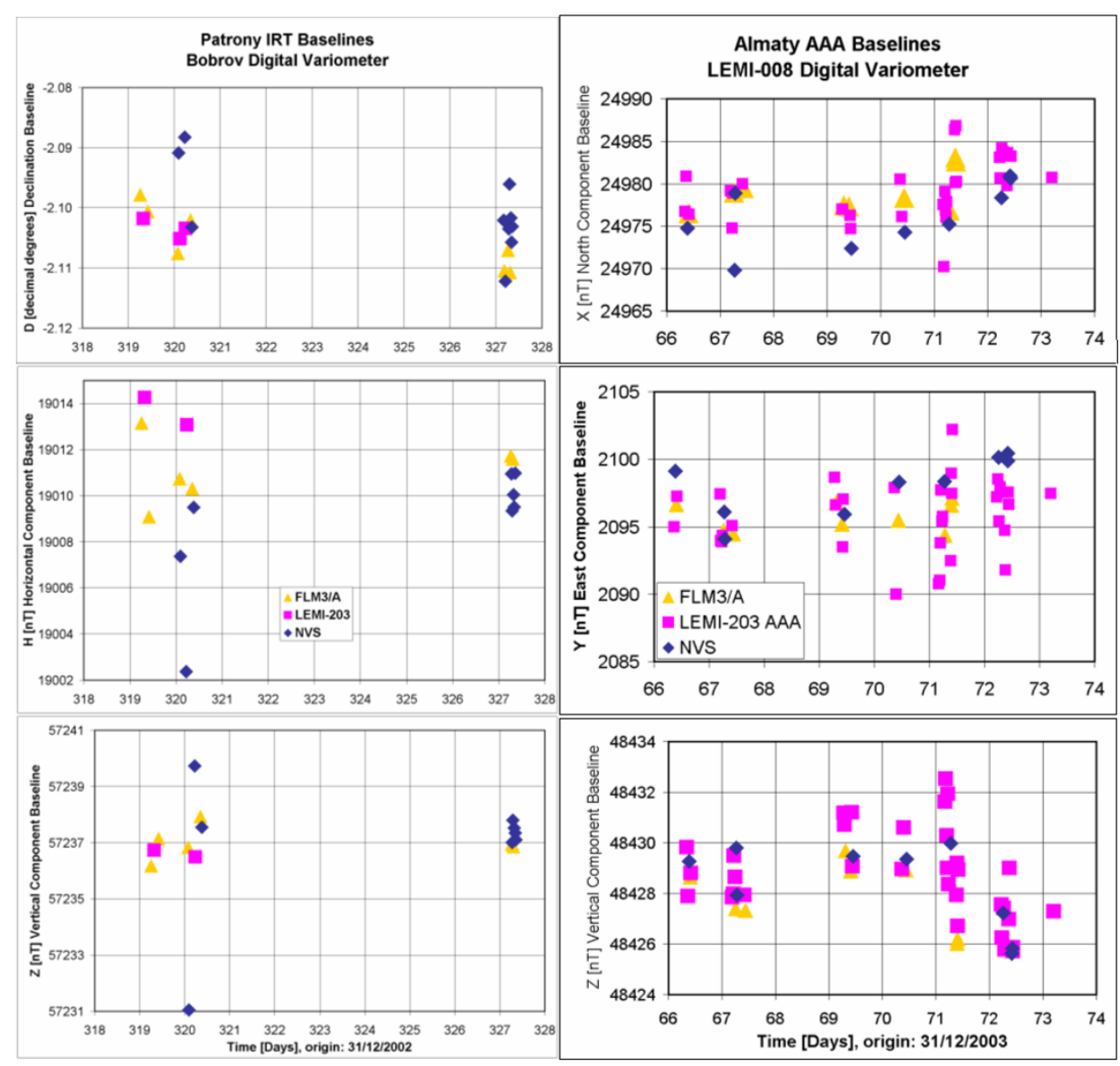

Fig. 3. Baselines obtained with CRENEGON DIfluxes during intercomparison sessions.

LCISR for the LEMI-008 and LEMI-203 in AAA and by the Observatories themselves elsewhere. Some buildings upgrade had to be made in order to accommodate the new equipment: stable non-magnetic pillars, cable ducts, security devices.

3.2 Calibration of the instrumentation and used standards

The standard used for the magnetic induction is the $K^{39}$ optically pumped self-oscillating scalar magnetometer
(Shifrin et al., 2000). This magnetometer claims an absolute accuracy of $0.2 \mathrm{nT}$. We calibrated an SMR90 Overhauser proton magnetometer in a coil system whose inner field is dynamically controlled by the $K^{39}$ (Rasson, 1996). This device is available at the Dourbes, Belgium magnetic observatory. Considering the calibration equation linking the true field $F$ to the magnetometer reading $F_{\mathrm{Mag}}$ via the 
Table 2. Results of calibration of POS-1 scalar magnetometers according to Eq. (1).

\begin{tabular}{cccc}
\hline & Arty ARS May 2003 & Irkutsk IRT and Uzur Nov 2003 & Almaty AAA March 2004 \\
\hline$E_{N 022}$ & $+0.01 \mathrm{nT}$ & & \\
$E_{N 100}$ & $+0.17 \mathrm{nT}$ & & \\
$E_{N 103}$ & $-0.04 \mathrm{nT}$ & $-0.04 \mathrm{nT}$ & $-0.29 \mathrm{nT}$ \\
$E_{N 108}$ & & & \\
$E_{N 117}$ & & $-0.00 \mathrm{nT}$ & $-0.17 \mathrm{nT}$ \\
$E_{N 118}$ & & & \\
\hline
\end{tabular}

magnetometer error $E_{\mathrm{Mag}}$ :

$$
F=F_{\mathrm{Mag}}-E_{\mathrm{Mag}}
$$

we get the result: $E_{\mathrm{SMR} 90}=+0.06 \mathrm{nT}$.

We used this magnetometer as a "travelling SMR90" to check and determine the error $E_{N \#}$ of all POS-1 Overhauser magnetometers purchased in the IA (Table 2). Thanks to its long cable $(30 \mathrm{~m})$ between electronic console and sensor, it was always possible to maintain the console-including the reference quartz oscillator-at room temperature. This was not possible for the POS-1, due to a shorter cable $(3 \mathrm{~m})$. Consequently, the POS-1 was sometimes calibrated with its console and quartz oscillator in very cold weather (down to $-20^{\circ} \mathrm{C}$ in Patrony). This affected the accuracy of calibrations as the temperature coefficient of the quartz oscillator should then be taken into account.

Another important device for the accuracy of magnetic vector measurements is the DIflux. This instrument for absolute measurements of declination and inclination, must answer to the following requirements in order to be considered as absolute (be an International Magnetic Standard IMS):

1. measure accurately angles ( $\sim 1$ second of arc),

2. be able to reference to the local vertical direction with at least the same accuracy and

3 . be free of ferromagnetism distorting the natural geomagnetic field direction by a measurable quantity.

DIfluxes are checked regularly by intercomparing them with those belonging to other observatories, as was done during this Kakioka workshop. Therefore we decided to use the FLM3/A ZEISS 010B DIflux as a "travelling FLM3/A" to intercompare all CRENEGON DIfluxes during the calibration and inspection visits to the targeted observatories. The FLM3/A was carefully intercompared at the Hermanus Workshop and at this Kakioka Workshop and found free of magnetic perturbation (requirement 3 ) while having a angle reading accuracy of better than 1 second of arc (requirement 1). The automatic levelling index based on a pendulum, allows referencing to the vertical with an accuracy of 0.3 seconds of arc (requirement 2). Requirement 3 can also be checked by approaching the DIflux from a high-sensitivity magnetometer and noting correlated deviations. It is worth to signal here that the LCSIR manufacturer tests its LEMI-203's DIfluxes in Belsk observatory for a full check of the device before delivery to the customers. This excellent practice is certainly a key step in the successful production of DIfluxes by LCSIR.

\subsection{Intercomparison of DIfluxes}

We had 3 meetings: Arty (Urals), Irkutsk (Siberia) and Almaty (Kazakhstan), for checking the accuracy of the LEMI-203 DIfluxes.

In Arty, Leonid Rakhlin from LCSIR participated in our meeting and brought with him the first LEMI-203 purchased by CRENEGON. We checked the non-magnetism of the LEMI-203 (after having removed the fluxgate sensor) with the Overhauser proton magnetometers in gradiometer configuration. No magnetic distortion could be found at the $0.1 \mathrm{nT}$ level with our measurement set-up.

In Irkutsk, at the Patrony Magnetic Observatory, an extensive check of various DIfluxes was organized, spanning several measurement sessions. Here the check was organized as in a workshop like this one in Kakioka, where the DIfluxes measure the baselines of a high stability variometer. Any distortion by the DIfluxes of the measured geomagnetic field will show up as deviations from the true baselines. The true baselines are given by the observer in charge and are confirmed if they correspond to the arithmetic means of the baselines measured by all participating DIfluxes - excluding any obvious outliers. In this case, special attention was paid to intercomparison with the FLM3/A. We find on Fig. 3 the baselines of the Bobrov digital variometer at Patrony IRT as measured by the FLM3/A and the CRENEGON LEMI-203 intended for IRT as well as another DIflux participating in the intercomparison sessions (NVS). The baselines of the LEMI and FLM3/A agree within $0.001^{\circ}$ for the declination, $2 \mathrm{nT}$ for $H$ and $0.2 \mathrm{nT}$ for the $Z$ baseline. Although the disagreement for $H$ is rather high, we believe it is due to an observer effect rather than to instrumental error. Indeed, magnetic pollution of the theodolite would mainly show up as a difference in the $Z$ baseline. And let's note that some of the observers performing in this intercomparison were not yet fully experienced ones. So one of the conclusions of the Irkutsk IRT meeting was that the first delivered LEMI-203 to CRENEGON was within the required accuracy specifications.

During the Almaty AAA meeting, one of the objectives was to fully intercompare the second LEMI-203 delivered by LCSIR to AAA. The travelling FLM3/A was available in AAA for that purpose. The procedure was again to measure the baselines of a stable variometer, in this case the LEMI-008 also delivered to AAA by LCSIR in the framework of CRENEGON. Details of the intercomparison are given in the Fig. 3. Although the dispersion in the 
Table 3. Renewed geomagnetic observatories of the NIS: equipment and INTERMAGNET status.

\begin{tabular}{|c|c|c|c|c|c|c|}
\hline & $\begin{array}{l}\text { LEMI-008/ } \\
\text { Variometer }\end{array}$ & $\begin{array}{c}\text { LEMI-203/ } \\
\text { other Abs. Instr. }\end{array}$ & POS-1 & PC/UPS & Transm. to GIN & IMO \\
\hline IRT, Irkutsk & $\checkmark$ & $\checkmark$ & $\mathrm{N} 103^{\dagger}, \mathrm{N} 117$ & 2 & $\checkmark$ & $\checkmark$ \\
\hline AAA, Almaty & $\checkmark$ & $\checkmark$ & N108, N118 & 2 & $\checkmark$ & \\
\hline NVS, Novosibirsk & $\checkmark$ & & & & $\checkmark$ & $\checkmark$ \\
\hline PET, Kamchatka & & $\checkmark$ & & & $\checkmark$ & \\
\hline KIV, Kiev & $\checkmark$ IPGP & $\checkmark$ & & & $\checkmark$ & \\
\hline BOX, Borok & VM391 IPGP & $\checkmark$ IPGP & & & $\checkmark$ & $\checkmark$ \\
\hline $\mathrm{LVV}^{*}, \mathrm{Lviv}$ & PSM Belsk & QHM declinometer & & & $\checkmark$ & $\checkmark$ \\
\hline
\end{tabular}

different determinations of the baseline by a single DIflux instrument is of the order of $3 \mathrm{nT}$-due to noisy magnetic conditions in the observatory-we can conclude that the $\mathrm{Y}$ baseline gives a difference between the AAA LEMI-203 and the FLM3/A not bigger than $0.5 \mathrm{nT}$. On inspection of the $X$ baseline, we can claim the same agreement level for $X$. According to the $Z$ baseline, there seems to be a difference of about $1 \mathrm{nT}$ between the 2 DIfluxes, indicating a possible small ferromagnetic contamination in the lower part of the LEMI-203. But again this could originate from the observers inexperience. Please also note that on day 71 , we found a small magnet located on the door of the absolute hut, serving as an intrusion detector. It was removed and this produced a noticeable step on the $X$ and $Z$ baselines. Finally we would like to point out that the AAA LEMI-203 participated in this Kakioka Workshop—as well as the NVS DIflux from Novosibirsk Klyuchi Magnetic Obsservatory. It is hence possible to check our intercomparisons with those performed in Kakioka.

\section{Training of the Observers and Documentation}

A good deal of training was planned early on in the project (Fig. 2). This training was organized in two ways: hands-on training and supply of documentation.

The hands-on training took place during the CRENEGON meetings and inspection visits by the coordinator. Existing or would-be observers were to perform absolute measurements under supervision during several sessions in their observatory absolute house after having been explained the full measurement protocol with the considered instrument, mainly the LEMI-203 DIflux. During this process, errors, tips and tricks for accurate and efficient operation would be pointed out to the person operating the instrument. Following the actual absolute geomagnetic measurements, a session of absolute data reduction was held on the data processing computer, where both the absolute and variometric data was processed and a reduction to the baseline of the variometer was performed.

The documentation consisted of

1. Instrument manuals: the LEMI-008, LEMI-203 and POS-1 manuals written in the Russian language.

2. A "GUIDE TO STATIONARY GEOMAGNETIC OBSERVATIONS" which was compiled by Stas Nechaev from Patrony observatory and edited by Jean Rasson.
This guide is available in both the English and Russian language. This 67 pages guide covers all modern aspects of Geomagnetic Observatory metrology, data storage and processing.

3. After the Almaty meeting it became clear that still some more documentation was necessary:

i. Proton magnetometer intercomparison techniques,

ii. Formulas demonstrating the absolute nature of the DIflux measurement protocol,

iii. Formulas for special spatial orientation of the variometer,

iv. Explicit formulas needed for a more in-depth understanding of the DIflux operation,

v. Field techniques for geomagnetic measurements with the DIflux.

Therefore a booklet "About Absolute Geomagnetic Measurements in the Observatory and in the Field" was compiled by J. Rasson.

\section{Evaluation of the Results: Baseline Plots of the Variometers}

A stable and well determined baseline for the observatory variometer is considered as one of the most pertinent quality checks for that observatory. The baseline in the ideal case of perfect variometer and absolute instruments and measurements should remain constant in an observatory measuring a homogeneous geomagnetic field. Baseline anomalies will hence directly show a measurement malfunction of variometer, absolute instruments or result from magnetic perturbations plaguing the observatory. The extent of the deviation of the baseline from a constant will indicate the importance of the malfunction. Due to lack of space we cannot display the baselines of IRT, AAA and NVS. In summary however, the baselines for IRT and NVS are OK, while there remains something to be done at AAA. Indeed IRT and NVS were accepted as IMO's by the INTERMAGNET consortium. We have had the promise of Dr. Valery Krasnov that in April 2005 he will take the necessary steps for improvement of the baselines in AAA. 


\section{Conclusion: Summary of Achieved Results}

In conclusion of this project, which terminated in Apri 2004, we are able to summarize the improvements in the infrastructure-both material and intellectual-of the observatories mentioned in Table 3. The original goal of 9 observatories could not be met due to budgetary restrictions imposed by the Action's funding. However we acknowledge the excellent support provided by INTAS through its Infrastructure Action IA-01-01.

Notes: * LVV was equipped by Belsk with a PSM (torsion photoelectric magnetometer) variometer, data logger, supply units, thermostat, along with specific IMO software and training.

† This magnetometer was destroyed in the fire of Patrony's variometer house on december 26th, 2003.

\section{References}

Jankowski, J. et al., Long-term stability of a torque-balance variometer with photoelectric converters in observatory practice, Geophysical Surveys, 6, 367-380, 1984.

Rasson, J. L., Coil system for the magnetic full field stabiliser, Proceedings of the VIth Workshop on Geomagnetic Observatory Instruments, Data Acquisition and Processing, edited by J. L. Rasson, Publ. Sci. et Techn., No. 003, Institut Royal Meteorologique de Belgique, Brussels, Belgium, pp. 87-93, 1996.

Shifrin, V. Ya., E. B. Alexandrov, T. I. Chikvadze, V. N. Kalabin, N. N. Yakobson, V. N. Khorev, and P. G. Park, Magnetic flux density standard for geomagnetometers, Metrologia, 37, 219-228, 2000.

J. L. Rasson (e-mail: jr@oma.be), A. S. Potapov, V. E. Korepanov, S. Y. Khomutov, V. M. Krasnov, and J. Bitterly 